\title{
Summary Report: Working Group 5 on "Electron Beam-Driven Plasma and Structure Based Acceleration Concepts"
}

\author{
Manoel E. Conde ${ }^{\dagger}$ and Thomas Katsouleas \\ ${ }^{\dagger}$ Argonne National Laboratory \\ High Energy Physics Division \\ 9700 S. Cass Ave. Bldg. 362 \\ Argonne, IL 60439 \\ "University of Southern California \\ Department of Electrical Engineering-Electrophysics \\ Los Angeles, CA 90089-0484
}

\begin{abstract}
The submitted manuscript has been created by the University of Chicago as Operator of Argonne National Laboratory ("Argonne") under Contract No. W-31-109-ENG-38 with the U.S. Department of Energy. The U.S Government retains for itself, and others acting on its behalf, a paid-up, nonexclusive. irrevocable worldwide license in said article to reproduce, prepare derivative works, distribute copies to the public, and perform publicly and display publicly, by or on behalf of the Government.
\end{abstract}

\begin{abstract}
The talks presented and the work performed on electron beam-driven accelerators in plasmas and structures are summarized. Highlights of the working group include new experimental results from the E-157 Plasma Wakefield Experiment, the E-150 Plasma Lens Experiment and the Argonne Dielectric Structure Wakefield experiments. The presentations inspired discussion and analysis of three working topics: electron hose instability, ion channel lasers and the plasma afterburner.
\end{abstract}

\section{INTRODUCTION}

This summary of the activities of the Working Group 5 is divided into two main parts: the first one giving a brief summary of the talks that were presented in the working group sessions, and the second part covering the material that was generated by small sub-groups that further explored the subject of some talks.

The talks were classified among the five following topics: Transverse Beam Dynamics and Radiation in Plasmas, PWFA (plasma wakefield accelerators) and Lens Experiments, Injection via Plasma Trapping (joint session with. Working Group 2), Structure Based Acceleration, and Ionization and Positron Acceleration. The topics that were discussed by the small sub-groups were: Hose Instability, Ion Channel Radiation, and Afterburner Design.

\section{SUMMARY OF TALKS}

The following tables list the talks that were presented at the working group sessions. Each table is followed by a brief summary of some of the talks. We apologize for not covering extensively all the talks and also for not listing the 


\section{DISCLAIMER}

This report was prepared as an account of work sponsored by an agency of the United States Government. Neither the United States Government nor any agency thereof, nor any of their employees, make any warranty, express or implied, or assumes any legal liability or responsibility for the accuracy, completeness, or usefulness of any information, apparatus, product, or process disclosed, or represents that its use would not infringe privately owned rights. Reference herein to any specific commercial product, process, or service by trade name, trademark, manufacturer, or otherwise does not necessarily constitute or imply its endorsement, recommendation, or favoring by the United States Government or any agency thereof. The views and opinions of authors expressed herein do not necessarily state or reflect those of the United States Government or any agency thereof. 


\section{DISCLAIMER}

Portions of this document may be illegible in electronic image products. Images are produced from the best available original document. 
speaker's co-workers (this was done for the sake of keeping this paper as just a summary; more information can, of course, be found in these proceedings). The talks that led to the discussions by small sub-groups are covered in the second part of this paper.

TABLE 1. Talks on Transverse Beam Dynamics and Radiation in Plasmas.

\begin{tabular}{lc}
\hline Presenter and affiliation & Title \\
\hline Patrick Muggli (USC) & Betatron Dynamics in E157 \\
Evan Dodd (UCLA) & Hosing Simulations \\
Brent Blue (UCLA) & Tail Oscillations in E157 \\
Sho Wang (UCLA) & Betatron X-Radiation in E157 and ICLs \\
Eric Esarey (LBNL) & Spontaneous Ion Channel Radiation Spectra and limits to ICL gain \\
\hline
\end{tabular}

All the talks above are related to two of the working sub-groups and are discussed later.

TABLE 2. Talks on PWFA and Lens Experiments.

\begin{tabular}{lcc}
\hline Presenter and affiliation & Title \\
\hline Mark Hogan (SLAC) & Optical Diagnostics in E157 (joint w/WG4) \\
Palma Catravas (LBNL) & Cerenkov Diagnosis of Plasma Density \\
Steve Russel (LANL) & LANL PWFA Observation (60 MeV/m deceleration) \\
Nick Barov (UCLA / FNAL) & PWFA Results at ANL and FNAL Plans & NOV \\
Patrick Muggli (USC) & $\sigma_{z}^{2}$ scaling of PWFA: proposed experiment at ATF & \\
Johnny Ng (SLAC) & E150 Plasma Lens Results \\
Pisin Chen (SLAC) & $100 \mathrm{GeV}$ PWFA & \\
\hline
\end{tabular}

Hogan presented an extensive discussion of the optical diagnostics used in the E157 experiment at SLAC, including the motivation for reflective optical elements used in pairs to cancel out aberrations. Catravas showed the measurements of Cerenkov radiation cones as a means to diagnose the plasma density and neutral gas density in the E-157 experiment. Russel presented preliminary results of the PWFA experiment at LANL that indicated a relatively high decelerating gradient. Barov showed acceleration of the electron bunch tail in his PWFA experiments at Argonne and the plans for a new series of experiments at Fermilab. Muggli presented the plans for experiments at Brookhaven to study the dependence of PWFA on bunch length.

J. Ng showed results from the plasma lens experiment at SLAC (E-150), indicating a reduction in the beam waist of about a factor two. This experiment was the first to realize the very high focusing gradients promised by plasma lens theory (order $\mathrm{GG} / \mathrm{m}$ ) and showed that plasma lenses also focus positrons. Chen showed alternative plans for the possibility of an Afterburner experiment at SLAC. The Afterburner concept aims at doubling the energy of a high energy beam in a single plasma wakefield accelerator stage and decreasing the spot size by a factor two.

TABLE 3. Talks on Injection via plasma trapping (w/ WG 2).

\begin{tabular}{lc}
\hline Presenter and affiliation & Title \\
\hline Christopher Moore (NRL) & Short pulse high-energy e- production from laser ionization \\
Ned Saleh (U. Mich.) & Recent results from U of Michigan \\
Hyyong Suk (UCLA) & Trapping at a density gradient in PWFA \\
\hline
\end{tabular}


cases with and without hose instability. The working sub-group generated a table with parameters for the electron beam and plasma for various experiments, and used the following asymptotic expression for the hose instability growth rate to predict whether hosing should be observed in the given experiments:

$$
N=\frac{3^{3 / 2}}{4}\left(k_{\beta} L\right)^{1 / 3}\left(\frac{\omega_{p} \sigma_{z}}{\sqrt{2} c}\right)^{2 / 3},
$$

where $k_{\beta}$ is the betatron wavenumber, $L$ is the length of the plasma column, $\omega_{p}$ is the plasma frequency, $\sigma_{z}$ is the bunch length, $c$ is the speed of light, and the growth rate of the instability is given by $\Gamma=e^{N}$.

TABLE 6. Electron Beam and Plasma Parameters, and expected Hose Instability Growth Rate

\begin{tabular}{lcccc}
\hline Parameter & $\begin{array}{c}\text { SLAC } \\
\text { design }\end{array}$ & $\begin{array}{c}\text { SLAC } \\
\text { "worst case" }\end{array}$ & ANL & FNAL \\
\hline$\sigma_{z}(\mathrm{~mm})$ & 0.6 & 1 & 6 & $0.5-5$ \\
$\sigma_{r}(\mu \mathrm{m})$ & 75 & 10 & 300 & 100 \\
$n_{\text {beam }}\left(\mathrm{cm}^{-3}\right)$ & $3.8 \times 10^{14}$ & $2.5 \times 10^{16}$ & $1.5 \times 10^{13}$ & $1.5 \times 10^{14}-1.5 \times 10^{15}$ \\
$n_{\text {plasma }}\left(\mathrm{cm}^{-3}\right)$ & $2.0 \times 10^{14}$ & $4.5 \times 10^{14}$ & $1.0 \times 10^{13}$ & $1.0 \times 10^{14}$ \\
$L(\mathrm{~m})$ & 1.4 & 1.4 & 0.12 & 0.08 \\
$\gamma$ & 60000 & 60000 & 28 & 30 \\
$N$ & 3.1 & 6.7 & 5.2 & 5.5 \\
\hline
\end{tabular}

The sub-group also examined the parameters used in Dodd's simulations and calculated the expected growth rate with the asymptotic expression. Surprisingly, the PIC simulations did not show hosing for some cases in which the asymptotic expression would predict instability. Apparently the PIC simulations indicated that hosing occurred when the asymptotic expression predicted it, but only if the beam density was sufficiently higher than the plasma density. This fact raises some concerns about the growth of this instability in a possible afterburner experiment, where the beam density may be approximately a thousand times higher than the plasma density.

The Ion Channel Radiation sub-group resulted from the discussion that followed the talks given by Wang and Esarey. The main topic under debate was the spectrum of the emitted radiation. Particularly, the consequences of the radial dependence of the betatron strength parameter. Some new ideas were brought up during the discussions: (1) to use a beam with small transverse dimension, minimizing the effect of the radial dependence of the betatron strength parameter; (2) to use a precursor beam with considerably larger transverse dimension, therefore creating a channel that would seem uniform to the smaller trailing beam; (3) to use a beam with an asymmetry in the transverse plane (slanted beam), therefore making use of the radial dependence of the radiation spectrum to create a chirped pulse.

The Afterburner Design sub-group considered the PWFA Afterburner design presented by Katsouleas in the plenary session, and discussed several issues related to the design of such an experiment. These included: (1) the possibility of increasing the total number of particles in the beams, in order to avoid using half of the present SLC 